\title{
Structure, Reactivity and Dynamics
}

\section{Foreword}

Understanding structure, reactivity and dynamics is the core issue in chemical sciences. This special volume presents a nice blend of contemporary theoretical and experimental research covering important aspects of density functional theory (DFT) calculations, molecular dynamics (MD) simulations, lightmolecule interactions, dynamics of chemical reactions including non-adiabatic interactions, collisions leading to reactions at cold and ultra-cold temperatures, development of theoretical methodologies and experimental techniques, and further strategies to deal with large-scale and nearly impossible computations. There are altogether 39 papers in this special issue contributed by leading researchers in the respective fields.

Density functional theoretical studies have been reported on hydrogen-bonded systems and their stabilities, chemical reactivity descriptors such as electronegativity and hardness, understanding interactions between two amino acids, proton affinity of amino acids in hydrophobic confinements, catalytic ability of certain organic reactions and adsorption processes.

First Principles MD simulations have been performed to study the dynamics of hydrogen bonding between water and protein oxygen atoms, the superionic conductors which exhibit both characteristics of liquids and solids, structure and transport studies of binary systems, dissociation of endo and exo 2-norbornyl chlorides and the effect of solvents on the dissociation processes.

The ultimate aim of a chemist is to develop the understanding and the ability to alter the rates of chemical reactions leading to desired products. During the last two decades two important themes, namely, the light-molecule interactions and molecular collisions at cold and ultra-cold (down up to milli-kelvins), have raised hopes in this directions. In the former case, by tuning the laser pulse in terms of strength, shape and time duration, one hopes to achieve the desired goal while in the latter case, one believes that quantum phenomena would be predominant leading to (sometimes counterintuitive!) desired products. Studies are reported on quantum dynamics of dissociation of a diatomic molecule in intense microwave laser, experiments involving single and multiphoton ionization of atomic clusters, theoretical investigations involving optical control of the irradiation of pericyclic reactions, the design of optimal laser pulse to control molecular rovibrational excitations, and experimental exploration of control parameters of the twophoton absorption processes in solutions for biologically relevant chromophores. Experiments involving laser-induced fluorescence spectroscopy to study van der Waal complexes and a brief account of ultrafast Raman Spectroscopy to study nonlinear processes are also presented. Quantum dynamics studies of chemical reactions in cold and ultra-cold environments are also reported.

The ubiquitous non-adiabatic processes involving the breakdown of the Born-Oppenheimer approximation are also studied. New and improved methodologies have been applied to study multi-surface multi-mode nuclear dynamics. The interesting phenomenon involving the geometric phase which may have important consequences on the dynamical attributes has been studied at conical intersections in a three-state Jahn-Teller system. Vibronic interactions at conical intersections and their effects on photoelectron spectra are reported.

There has been a renewed interest in the study of low-energy electron-molecule collisions in recent years particularly in view of the formation and decay of the resonant metastable negative molecular ion which can decay either giving rise to internally excited neutral molecule plus (an extra) electron or dissociate into the neutral and anionic fragments (disssociative electron attachment, DEA). Quantum mechanical time-dependent wave packet studies have been undertaken to study the vibrational excitations of molecules. New experimental results on the DEA in $\mathrm{H}_{2} \mathrm{O}$ and $\mathrm{D}_{2} \mathrm{O}$ are also presented. 
Many other important structural and dynamics studies are also reported: quantum dynamics of intermolecular $\mathrm{H}$-atom transfer leading to isomerization by tunnelling, multiple proton transfer reactions, dynamical transition between two entropy states associated with the shape change of the confinement of a stochastic system applied to liquid-gas phase transition, quantum dynamics of bimolecular reaction, new strategy to obtain partition function of the two-dimensional nearest neighbours using Ising Model for finite lattices in a non-zero magnetic field, theoretical studies on excitation energy transfer from a dye molecule to doped graphene, hydrogen adsorption properties of silicon-lithium binary clusters, and mechanism of intermolecular vibrational energy redistribution in highly vibrationally excited molecules.

Further developments are reported in theoretical ab initio tools involving the multi-reference coupledcluster approach to include triple excitations and applied to the dipole moment calculations which are known to be quite sensitive to electron correlation. Some new theoretical insights are presented on general properties for the bound states. Their dimensionality and scaling lead to interesting properties of the uncertainty product, entropies and information. Theoretical implications are also discussed on the simple connection between the Slater orbitals and the Coulomb Sturmian orbitals and the relationships between the two basis sets.

In view of large scale computations, new insights such as tailoring approach for obtaining molecular orbitals of large systems is discussed. A brief account is presented on the development of the 'Bhageerath' - an ab initio model to study protein folding with MD simulations, which predicts protein tertiary structures. These papers highlight the computational achievements of the near impossible category!

We would like to express our sincere thanks and appreciation to all the authors and the reviewers. We also thank the Editor, and the Editorial Board of Journal of Chemical Sciences for agreeing to bring out this special issue. Our sincere thanks to Mr. T D Mahabaleswara and Ms N Padmaja for their help and editorial support to bring out this special issue.

3 January 2012

SUSANTA MAHAPATRA

School of Chemistry, University of Hyderabad, Hyderabad 500 046, India e-mail: smsc@uohyd.ernet.in

SANJAY KUMAR Department of Chemistry, Indian Institute of Technology Madras,

Chennai 600 036, India

e-mail: sanjay@iitm.ac.in

(Guest Editors) 